\title{
The stable equivalence and cancellation problems
}

\author{
Leonid Makar-Limanov*, Peter van Rossum, Vladimir Shpilrain and Jie-Tai Yu ${ }^{\dagger}$
}

\begin{abstract}
Let $K$ be an arbitrary field of characteristic 0 , and $\mathbf{A}^{n}$ the $n$-dimensional affine space over $K$. A well-known cancellation problem asks, given two algebraic varieties $V_{1}, V_{2} \subseteq \mathbf{A}^{n}$ with isomorphic cylinders $V_{1} \times \mathbf{A}^{1}$ and $V_{2} \times \mathbf{A}^{1}$, whether $V_{1}$ and $V_{2}$ themselves are isomorphic.

In this paper, we focus on a related problem: given two varieties with equivalent (under an automorphism of $\mathbf{A}^{n+1}$ ) cylinders $V_{1} \times \mathbf{A}^{1}$ and $V_{2} \times \mathbf{A}^{1}$, are $V_{1}$ and $V_{2}$ equivalent under an automorphism of $\mathbf{A}^{n}$ ? We call this stable equivalence problem. We show that the answer is positive for any two curves $V_{1}, V_{2} \subseteq \mathbf{A}^{2}$

For an arbitrary $n \geq 2$, we consider a special, arguably the most important, case of both problems, where one of the varieties is a hyperplane. We show that a positive solution of the stable equivalence problem in this case implies a positive solution of the cancellation problem.
\end{abstract}

Mathematics Subject Classification (2000). Primary 14E09, 14E25; Secondary 14A10, $13 \mathrm{~B} 25$.

Keywords. Algebraic varieties, cancellation problem, polynomial automorphisms, stable equivalence, Danielewski surfaces.

\section{Introduction}

Let $K\left[x_{1}, \ldots, x_{n}\right]$ be the polynomial algebra in $n$ variables over a field $K$ of characteristic 0 . Any collection of polynomials $p_{1}, \ldots, p_{m}$ from $K\left[x_{1}, \ldots, x_{n}\right]$ determines an algebraic variety $\operatorname{Spec} K\left[x_{1}, \ldots, x_{n}\right] /\left\langle p_{1}, \ldots, p_{m}\right\rangle$ in the affine space $\mathbf{A}^{n}=\mathbf{A}_{K}^{n}=\operatorname{Spec} K\left[x_{1}, \ldots, x_{n}\right]$. If $K$ is algebraically closed and $\left\langle p_{1}, \ldots, p_{m}\right\rangle$ is radical, we can of course think of this variety as the zero set $\left\{p_{i}=0, i=1, \ldots, m\right\}$ in $K^{n}$. We denote this algebraic variety by $V\left(p_{1}, \ldots, p_{m}\right)$.

We say that two algebraic varieties $V\left(p_{1}, \ldots, p_{m}\right)$ and $V\left(q_{1}, \ldots, q_{k}\right)$ in $\mathbf{A}^{n}$ are equivalent if there is an automorphism of $\mathbf{A}^{n}$ that takes one of them onto the other. Algebraically, this means there is an automorphism of $K\left[x_{1}, \ldots, x_{n}\right]$ that takes the ideal $\left\langle p_{1}, \ldots, p_{m}\right\rangle$ to the ideal $\left\langle q_{1}, \ldots, q_{k}\right\rangle$.

A variety equivalent to $V \times \mathbf{A}^{1}$ is called a cylinder; a variety of the form

*Partially supported by an NSA grant.

$\dagger$ †) Partially supported by Hong Kong RGC-CERG Grants 10203186 and 10203669. 
$V(p)$ is called a hypersurface, and a hypersurface equivalent to $V\left(x_{1}\right)$ is called a hyperplane.

We say that two algebraic varieties $V_{1}$ and $V_{2}$ in $\mathbf{A}^{n}$ are stably equivalent if there is an automorphism of $\mathbf{A}^{m}$ for some $m>n$ that takes the cylinder $V_{1} \times \mathbf{A}^{m-n}$ onto $V_{2} \times \mathbf{A}^{m-n}$.

We also say that two polynomials $p, q \in K\left[x_{1}, \ldots, x_{n}\right]$ are stably equivalent if $\alpha(p)=q$ for some automorphism $\alpha$ of $K\left[x_{1}, \ldots, x_{m}\right], m>n$.

We address here the following

Stable equivalence problem. Is it true that stable equivalence of two hypersurfaces in $\mathbf{A}^{n}$ implies their equivalence?

Or, in purely algebraic language: are any two stably equivalent polynomials equivalent?

If one considers arbitrary algebraic varieties, not just hypersurfaces, then the answer is negative, as explained in [17]. The corresponding example is based on a well-known example, due to Danielewski, of non-isomorphic surfaces in $\mathbf{C}^{3}$ with isomorphic cylinders.

Here we solve the Stable equivalence problem for $n=2$ :

Theorem 1.1. If two polynomials $p, q \in K[x, y]$ are stably equivalent, then they are equivalent. Or, in geometric language: if $V(p)$ and $V(q)$ are two curves in $\mathbf{A}^{2}$ such that, for some $s \geq 1$, the cylinders $V(p) \times \mathbf{A}^{s}$ and $V(q) \times \mathbf{A}^{s}$ are equivalent in $\mathbf{A}^{2+s}$, then $V(p)$ and $V(q)$ are equivalent in $\mathbf{A}^{2}$.

Upon replacing equivalence with isomorphism in the statement of Theorem 1.1, one gets a well known result of Abhyankar, Eakin and Heinzer [1].

Now we focus on a special case of the Stable equivalence problem; we call it

Stable coordinate conjecture. Let $V(p)$ be a hypersurface in $\mathbf{A}^{n}$. If $V(p) \times \mathbf{A}^{1}$ is equivalent to a hyperplane in $\mathbf{A}^{n+1}$, then $V(p)$ is equivalent to a hyperplane in $\mathbf{A}^{n}$.

Or, in purely algebraic language: if $p=p\left(x_{1}, \ldots, x_{n}\right)$ and $\varphi(p)=x_{1}$ for some automorphism $\varphi$ of $K\left[x_{1}, \ldots, x_{n+1}\right]$, then also $\alpha(p)=x_{1}$ for some automorphism $\alpha$ of $K\left[x_{1}, \ldots, x_{n}\right]$, i.e., $p$ is a coordinate in $K\left[x_{1}, \ldots, x_{n}\right]$.

It turns out that the Stable coordinate conjecture is closely related to the famous Cancellation conjecture of Zariski:

Cancellation conjecture. Let $V(p)$ be a hypersurface in $\mathbf{A}^{n}$. If $V(p) \times \mathbf{A}^{1}$ is isomorphic to a hyperplane in $\mathbf{A}^{n+1}$ (i.e., to $\mathbf{A}^{n}$ ), then $V(p)$ is isomorphic to a hyperplane in $\mathbf{A}^{n}$ (i.e., to $\mathbf{A}^{n-1}$ ).

Or, in purely algebraic language: if, for some $K$-algebra $R, R[x]$ is isomorphic to $K\left[x_{1}, \ldots, x_{n}\right]$, then $R$ is isomorphic to $K\left[x_{1}, \ldots, x_{n-1}\right]$. 
This conjecture was proved for $n=2$ [1], [12] and $n=3$ [13], [9]. There is some circumstantial evidence that it might be wrong in higher dimensions if $K=\mathbf{R}$, see [3]. We refer to [10] for a more detailed survey on this problem.

In [17], it was shown that, for each particular $n$, the Cancellation conjecture follows from the Stable coordinate conjecture combined with the Embedding conjecture of Abhyankar and Sathaye (see [2]), and also that the Stable coordinate conjecture follows from the Cancellation conjecture combined with the Embedding conjecture.

Here we establish a more straightforward implication:

Theorem 1.2. For each particular n, the Stable coordinate conjecture implies the Cancellation conjecture.

It would be interesting to pinpoint also some connection between more general forms of both conjectures, namely, between what we call the Stable equivalence problem and the Cancellation problem (see the abstract). In particular, having in mind Danielewski's example mentioned before and motivated by Theorem 1.2, we ask:

Problem 1. Let $p=p(x, y, z)=x y+z^{2}$. Is it true that every polynomial in $K[x, y, z]$ which is stably equivalent to $p$ is, in fact, equivalent to $p$ ?

Recall that, by results of Danielewski [5] and Fieseler [7], the hypersurface $D(k)=\left\{x y^{k}+z^{2}+1=0\right\}$ is not isomorphic to $D(m)=\left\{x y^{m}+z^{2}+1=0\right\}$ if $k \neq m, k, m \geq 1$, whereas the cylinders $D(k) \times \mathbf{C}$ and $D(m) \times \mathbf{C}$ are isomorphic.

Finally, we mention that it would be also interesting to find any relation between the general Cancellation problem and the general Embedding problem. A somewhat bold conjecture would be that if, for a hypersurface $V(p) \subseteq \mathbf{A}^{n}$, the cylinder $V(p) \times \mathbf{A}^{1}$ has a unique (up to an automorphism of $\mathbf{A}^{n+1}$ ) embedding into $\mathbf{A}^{n+1}$, then, whenever $V(p) \times \mathbf{A}^{1}$ is isomorphic to $V(q) \times \mathbf{A}^{1}$, one has $V(p)$ isomorphic to $V(q)$.

Now a natural question is whether or not Danielewski's surfaces/cylinders have unique embeddings in $\mathbf{C}^{4}$. We were able to prove that all but one of them do not:

Proposition 1.3. For any $m \geq 2$, the hypersurface $D(m) \times \mathbf{C}^{k-3}=\left\{x y^{m}+z^{2}+\right.$ $1=0\}$ has at least 2 inequivalent embeddings in $\mathbf{C}^{k}$ for any $k \geq 3$.

We note that for $k=3$, this was also proved in [8] (by an altogether different method). For $m=1$, the question is open:

Problem 2. Does the hypersurface $D(1) \times \mathbf{C}=\left\{x y+z^{2}+1=0\right\}$ have a unique embedding in $\mathbf{C}^{4}$ ? 
It is also unknown whether $D(1)$ has a unique embedding in $\mathbf{C}^{3}$ (cf. [8, Question 1]).

We note that Problems 1 and 2 cannot both have positive answers. Indeed, if the answer to Problem 2 was positive, then, since we know that $D(1) \times \mathbf{C}$ is isomorphic to $D(m) \times \mathbf{C}$ for any $m \geq 1$, we would have $D(1) \times \mathbf{C}$ equivalent to $D(m) \times \mathbf{C}$ in $\mathbf{C}^{4}$. Then, if the answer to Problem 1 was positive, this would imply that $D(1)$ is equivalent to $D(m)$ in $\mathbf{C}^{3}$, which is known not to be the case.

This simple trick also works in a more general situation, namely:

Proposition 1.4. Let $V(p), p=p\left(x_{1}, \ldots, x_{n}\right)$, be a hypersurface in $\mathbf{A}^{n}$. Suppose that the following two conditions hold:

(i) $V(p) \times \mathbf{A}^{1}$ has a unique embedding in $\mathbf{A}^{n+1}$.

(ii) If $V(p)$ is stably equivalent to $V(q)$ for some $q=q\left(x_{1}, \ldots, x_{n}\right)$, then $V(p)$ is equivalent to $V(q)$.

Then, whenever $V(p) \times \mathbf{A}^{1}$ is isomorphic to $V(q) \times \mathbf{A}^{1}$, it follows that $V(p)$ and $V(q)$ are isomorphic subvarieties of $\mathbf{A}^{n}$.

The proof is obvious; we omit the details. Equally obvious is the following

Proposition 1.5. Let $V(p), p=p\left(x_{1}, \ldots, x_{n}\right)$, be a hypersurface in $\mathbf{A}^{n}$. Suppose that the following two conditions hold:

(i) $V(p)$ has a unique embedding in $\mathbf{A}^{n}$.

(ii) If $V(p) \times \mathbf{A}^{s}$ and $V(q) \times \mathbf{A}^{s}$ are isomorphic subvarieties of $\mathbf{A}^{n+s}$ for some $s \geq 1$, then $V(p)$ and $V(q)$ are isomorphic subvarieties of $\mathbf{A}^{n}$.

Then, whenever $V(p)$ is stably equivalent to $V(q)$ for some $q=q\left(x_{1}, \ldots, x_{n}\right)$, one has $V(p)$ equivalent to $V(q)$.

\section{The two-variable case}

Let $p, q \in K[x, y], \varphi(p)=q$ for some automorphism $\varphi$ of $K[x, y, z, \ldots]$.

Let $\varphi(x)=u=u(x, y, z, \ldots), \varphi(y)=v=v(x, y, z, \ldots)$. We are going to prove a stronger statement (Proposition 2.1 below) that will imply Theorem 1.1.

We call a pair $(u, v)$ of polynomials $z$-reduced if the sum of $z$-degrees of the two polynomials cannot be reduced by either a (non-degenerate) linear transformation or a transformation of one of the following two types:

(i) $(u, v) \longrightarrow\left(u+\mu \cdot v^{k}, v\right)$ for some $\mu \in K^{*} ; k \geq 2$;

(ii) $(u, v) \longrightarrow\left(u, v+\mu \cdot u^{k}\right)$.

When proving Theorem 1.1, we can assume, without loss of generality, that the pair $(u(x, y, z, \ldots), v(x, y, z, \ldots))$ is $z$-reduced.

If $(u, v)$ is a pair of two-variable polynomials such that the sum of their degrees cannot be reduced by a transformation of one the above types, then we call this pair elementary reduced. 
Proposition 2.1. Let $p \in K[x, y]$ be a two-variable polynomial. Let $(u(x, y, z, \ldots)$, $v(x, y, z, \ldots))$ be a $z$-reduced pair of algebraically independent polynomials such that both of them actually depend on $z$. Then, for any $N \in \mathbf{Z}_{+}$, there is a polynomial $w=w(x, y)$ such that $\operatorname{deg}\left(p\left(u\left(x, y, w, c_{1}, c_{2}, \ldots\right), v\left(x, y, w, c_{1}, c_{2}, \ldots\right)\right)\right)>N$, for some choice of constants $c_{1}, c_{2}, \ldots$

In the proof of Proposition 2.1, we shall write just $u(x, y, z)$ and $v(x, y, z)$ instead of $u\left(x, y, z, c_{1}, c_{2}, \ldots\right)$ and $v\left(x, y, z, c_{1}, c_{2}, \ldots\right)$ to simplify the notation. First we prove

Lemma 2.2. Let $u(x, y, z)$ and $v(x, y, z)$ be algebraically independent. For any $M \in \mathbf{Z}_{+}$and $m, n>M$, there is $c \in K$ such that $u\left(x, y, x^{m} y^{n}+c\right)$ and $v\left(x, y, x^{m} y^{n}+c\right)$ are algebraically independent.

Proof. Recall that polynomials $f_{1}, \ldots, f_{m} \in K\left[x_{1}, \ldots, x_{n}\right]$ are algebraically dependent over $K$ if and only if the Jacobian matrix $D\left(f_{1}, \ldots, f_{m}\right)$ has rank smaller than $m$.

Assume, by way of contradiction, that for all $c \in K$ the polynomials $u\left(x, y, x^{m} y^{n}+c\right), v\left(x, y, x^{m} y^{n}+c\right) \in K[x, y]$ are algebraically dependent. This means that for all $c \in K$, the matrix

$$
\begin{aligned}
& D\left(u\left(x, y, x^{m} y^{n}+c\right), v\left(x, y, x^{m} y^{n}+c\right)\right) \\
& =\left.D(u, v)\right|_{\left(x, y, x^{m} y^{n}+c\right)} \cdot\left(\begin{array}{cc}
1 & 0 \\
0 & 1 \\
m x^{m-1} y^{n} & n x^{m} y^{n-1}
\end{array}\right)
\end{aligned}
$$

has rank at most 1 . Then for all $c \in K$ the $2 \times 3$ matrix $\left.D(u, v)\right|_{\left(x, y, x^{m} y^{n}+c\right)}$ has rank at most one, which means that all its $2 \times 2$ minors are 0 . Using the fact that for all $a, b \in K$ the map from $K$ to $K$ definied by $c \mapsto a^{m} b^{n}+c$ is surjective, this implies that for all $a, b, c \in K$ all $2 \times 2$ minors of $\left.D(u, v)\right|_{(a, b, c)}$ are 0 . Since $K$ is an infinite field, this in turn implies that all $2 \times 2$ minors of $D(u, v)$ are 0 . Thus, the rank of $D(u, v)$ is at most one and therefore $u$ and $v$ are algebraically dependent over $K$, a contradiction.

Proof of Proposition 2.1. Since both $u(x, y, z)$ and $v(x, y, z)$ actually depend on $z$ and the pair is $z$-reduced, we can find $m, n>2 N$ and $c \in K$ such that $u\left(x, y, x^{m} y^{n}+c\right)$ and $v\left(x, y, x^{m} y^{n}+c\right)$ are algebraically independent (by Lemma 2.2) and elementary reduced.

Now we use a result of Shestakov and Umirbaev [15] which implies, in particular, that, if two polynomials $r(x, y)$ and $s(x, y)$ of degree $>2 N$ are algebraically independent and elementary reduced, then every non-constant polynomial in the algebra $K[r, s]$ has degree at least $N+2$. This completes the proof of Proposition 2.1 .

Now we can get to the 
Proof of Theorem 1.1. Recall that $\varphi(x)=u=u(x, y, z, \ldots), \varphi(y)=v=$ $v(x, y, z, \ldots)$. Upon applying an automorphism of $K[x, y]$ to both $p(x, y)$ and $q(x, y)$ if necessary, we may assume that $u(x, y, z, \ldots)$ and $v(x, y, z, \ldots)$ are $z$ reduced. Now we have several cases.

Case 1. Both $u(x, y, z, \ldots)$ and $v(x, y, z, \ldots)$ actually depend on $z$. Then we can apply Proposition 2.1 to get a contradiction in this case.

Case 2. Say, $v(x, y, z, \ldots)$ actually depends on $z$, whereas $u(x, y, z, \ldots)$ does not. Let $x^{m} y^{n}$ be the highest monomial in $p(x, y)$ with respect to "lexdeg" ordering with $y>x$. This monomial will contain the highest power of $z$ after we plug in $u$ for $x$ and $v$ for $y$. This highest power of $z$ then cannot cancel out in $p(u, v)$. Therefore, $p(u, v)$ will depend on $z$, contrary to the assumption $p(u, v)=q(x, y)$.

Case 3. Neither $u(x, y, z, \ldots)$ nor $v(x, y, z, \ldots)$ depend on $z$. If there are other variables that either $u(x, y, z, \ldots)$ or $v(x, y, z, \ldots)$ depend on, then we find ourselves in Case 1 or 2 above. If not, then there is nothing to prove because the restriction of $\varphi$ to $K[x, y]$ must be an automorphism of $K[x, y]$.

This completes the proof of Theorem 1.1.

Remark. The crucial technical tool in our proof of Theorem 1.1 was ShestakovUmirbaev's result from [15] that bounds (from below) the degree of polynomials in the subalgebra of $K[x, y]$ generated by two given polynomials. This is (philosophically) similar to "small cancellation" ideas in combinatorial group theory (see e.g. [11]). We note however that in commutative algebra, these ideas cannot be simply carried on to higher dimensions as the following example shows.

Let $\varphi: x \rightarrow u=x-y t^{2} z^{2}, y \rightarrow v=1+t z^{2}, z \rightarrow r=z^{2}, t \rightarrow s=-x t+y t^{2}+$ $y t^{3} z^{2}$. Let $p=p(x, y, z, t)=x y+z t$. Then $\varphi(p)=x$, i.e., $x \in K[u, v, r, s]$, even though the degrees of $u, v, r, s$ are at least 2. Similar examples can be constructed with arbitrarily high degrees of $u, v, r, s$.

This example therefore makes it appear likely that our proof of Theorem 1.1 might be difficult to carry on to higher dimensions, but, of course, this does not mean that the result itself does not hold.

Finally, since our proof of Theorem 1.1 heavily relies on Shestakov-Umirbaev's result which is not yet published, we offer an alternative proof, which is more elementary, but probably has more limited use.

Alternative proof of Theorem 1.1. The statement will follow from Proposition 2.3 below.

Proposition 2.3. Let $R=K\left[x_{1}, \ldots, x_{n}, z\right]$ be a ring of polynomials in $(n+1)$ variables and let $(u, v)$ be a z-reduced pair of algebraically independent polynomials from $R$ such that both of them actually depend on $z$. Then, for any nonconstant two-variable polynomial $p$, the polynomial $p(u, v)$ depends on $z$, too. 
Proof. Suppose, by way of contradiction, that $q=p(u, v) \in K\left[x_{1}, \ldots, x_{n}\right]$. It is clear that $\frac{\partial q}{\partial x} \neq 0$ for some $x=x_{i}$ : otherwise $q$ would be a constant, and $u$ and $v$ would be algebraically dependent, contrary to the assumption.

Then the derivation $\partial(g)=J_{x, z}(q, g)=\frac{\partial q}{\partial x} \frac{\partial g}{\partial z}-\frac{\partial q}{\partial z} \frac{\partial g}{\partial x}=\frac{\partial q}{\partial x} \frac{\partial g}{\partial z}$ is a nonzero locally nilpotent derivation on $R$, i.e., for any element $g \in R$ there is $n$ such that $\partial^{n}(g)=0$. Indeed, since $\operatorname{deg}_{z} q=0$, we see that $\operatorname{deg}_{z} \partial(g)<\operatorname{deg}_{z} g$. Therefore, if $m=\operatorname{deg}_{z} g$, then $\partial^{m+1}(g)=0$.

Define now a derivation $\partial_{1}$ on $K[u, v] \subset R$ as follows: $\partial_{1}(f)=J_{u, v}(p(u, v), f)$ for $f=f(u, v) \in K[u, v]$. We claim that this derivation is locally nilpotent, too. Let $g=g\left(x_{1}, \ldots, x_{n}, z\right)=f(u, v) \in K[u, v]$. By the usual chain rule, we have $\partial(g)=J_{x, z}(q, g)=J_{x, z}(p(u, v), f(u, v))=J_{x, z}(u, v) \cdot J_{u, v}(p, f)=J_{x, z}(u, v) \cdot \partial_{1}(f)$. Thus, if we consider $\partial_{1}(f)$ as an element of $R$ (as opposed to just $K[u, v]$ ), then $\operatorname{deg}_{z} \partial_{1}(f)=\operatorname{deg}_{z} \partial(g)-\operatorname{deg}_{z} J_{x, z}(u, v)<\operatorname{deg}_{z} g=\operatorname{deg}_{z} f(u, v)$. As above, this implies that $\partial_{1}$ is a locally nilpotent derivation on $K[u, v]$ since every application of $\partial_{1}$ decreases the degree relative to $z$.

Locally nilpotent derivations on a polynomial ring in two variables are well understood. In particular, it is known that the kernel of a nonzero locally nilpotent derivation is a polynomial ring in one variable and its generator is also a generator of the ambient two-variable ring (see [14]). Since $\partial_{1}$ is a nonzero derivation and $p \in \operatorname{ker} \partial_{1}$, a generator $s$ of the kernel does not depend on $z$ either. Thus, $K[u, v]=$ $K[s, w]$, where $s$ does not depend on $z$, and therefore $\operatorname{deg}_{z} u=\operatorname{deg}_{w} u \cdot \operatorname{deg}_{z} w$, $\operatorname{deg}_{z} v=\operatorname{deg}_{w} v \cdot \operatorname{deg}_{z} w$. It is known (see e.g. [4]) that $K[u, v]=K[s, w]$ implies that either $\operatorname{deg}_{w} u$ divides $\operatorname{deg}_{w} v$ or $\operatorname{deg}_{w} v$ divides $\operatorname{deg}_{w} u$ and that there is an elementary transformation (see the beginning of this section) which reduces the $w$ degree of the pair $(u, v)$. Therefore, the $z$-degree of the pair can be reduced, too, so that $(u, v)$ is not a $z$-reduced pair contrary to our assumption. This completes the proof.

\section{The Stable coordinate and other conjectures}

Proof of Theorem 1.2. One of the equivalent formulations of the Cancellation conjecture is (see [6, p. 54]): for every locally nilpotent derivation $\mathcal{D}$ of the algebra $K\left[x_{1}, \ldots, x_{n}\right]$ with a slice $s$, the kernel $\operatorname{Ker} \mathcal{D}$ is isomorphic to $K\left[x_{1}, \ldots, x_{n-1}\right]$. By Proposition 2.1 of Wright [18], the latter property is equivalent to $s$ being a coordinate, i.e., an automorphic image of $x_{1}$.

Thus, we start with an arbitrary locally nilpotent derivation $\mathcal{D}$ of the algebra $K\left[x_{1}, \ldots, x_{n}\right]$ with a slice $s$, and we want to prove that $s$ is a coordinate in $K\left[x_{1}, \ldots, x_{n}\right]$.

Extend $\mathcal{D}$ to $K\left[x_{1}, \ldots, x_{n+1}\right]$ by $\mathcal{D}\left(x_{n+1}\right)=0$. Then $\operatorname{Ker} \mathcal{D}$ in $K\left[x_{1}, \ldots, x_{n+1}\right]$ is $K\left[x_{1}, \ldots, x_{n}\right]^{\mathcal{D}}\left[x_{n+1}\right]$, where $K\left[x_{1}, \ldots, x_{n}\right]^{\mathcal{D}}$ denotes $K \operatorname{Ker} \mathcal{D}$ in $K\left[x_{1}, \ldots, x_{n}\right]$. Since $s$ is transcendental over $K\left[x_{1}, \ldots, x_{n}\right]^{\mathcal{D}}$, we have $K\left[x_{1}, \ldots, x_{n}\right]^{\mathcal{D}}\left[x_{n+1}\right]$ iso- 
morphic to $K\left[x_{1}, \ldots, x_{n}\right]^{\mathcal{D}}[s]$. The latter algebra is equal to $K\left[x_{1}, \ldots, x_{n}\right]$ by the result of Wright [18, Proposition 2.1].

Thus we get $K\left[x_{1}, \ldots, x_{n+1}\right]^{\mathcal{D}}$ isomorphic to $K\left[x_{1}, \ldots, x_{n}\right]$, which implies that $s$ is a coordinate in $K\left[x_{1}, \ldots, x_{n+1}\right]$. Since we are under the assumption that the Stable coordinate conjecture holds for this particular $n$, we conclude that $s$ is a coordinate in $K\left[x_{1}, \ldots, x_{n}\right]$, and therefore the Cancellation conjecture holds for the same $n$.

Proof of Proposition 1.3. We give a proof here for $m=2$, just to simplify the notation. As in [16], it will be technically more convenient to write algebras of residue classes as "algebras with relations", i.e., for example, instead of $K\left[x_{1}, \ldots, x_{n}\right] /\left\langle p\left(x_{1}, \ldots, x_{n}\right)\right\rangle$ we shall write $\left\langle x_{1}, \ldots, x_{n} \mid p\left(x_{1}, \ldots, x_{n}\right)=0\right\rangle$.

We get the following chain of "elementary" isomorphisms:

$\left\langle x, y, z \mid x y^{2}+z^{2}+1=0\right\rangle \cong$ (applying the automorphism $\phi: x \rightarrow x, y \rightarrow$ $y+1, z \rightarrow z)\left\langle x, y, z \mid x=-x y^{2}-2 x y-z^{2}-1\right\rangle \cong\langle x, y, z, u| u=x y, x=$ $\left.-u y-2 u-z^{2}-1\right\rangle \cong\left\langle x, y, z, u \mid u=-u y^{2}-2 u y-z^{2} y-y, x=-u y-2 u-z^{2}-1\right\rangle \cong$ $\left\langle y, z, u \mid u=-u y^{2}-2 u y-z^{2} y-y\right\rangle \cong\left\langle x, y, z \mid x=-x y^{2}-2 x y-z^{2} y-y\right\rangle \cong$ (applying the automorphism $\phi: x \rightarrow x, y \rightarrow y-1, z \rightarrow z)\left\langle x, y, z \mid x y^{2}+z^{2} y-z^{2}+y-1=0\right\rangle$.

Now let $p=p(x, y, z)=x y^{2}+z^{2}+1, q=q(x, y, z)=x y^{2}+z^{2} y-z^{2}+y-1$. We are going to show that the $\operatorname{gradients} \operatorname{grad}(p)$ and $\operatorname{grad}(q)$ have different numbers of zeros. This obviously implies that $p$ and $q$ are inequivalent under any automorphism of $K[x, y, z]$ (in fact, this implies that $p$ and $q$ are even stably inequivalent).

Compute:

$$
\begin{gathered}
\operatorname{grad}(p)=\left(y^{2}, 2 x y, 2 z\right) \\
\operatorname{grad}(q)=\left(y^{2}, 2 x y+z^{2}+1,2 y z-2 z\right) .
\end{gathered}
$$

We see that $\operatorname{grad}(p)$ has infinitely many zeros $(y=z=0, x$ arbitrary), whereas $\operatorname{grad}(q)$ has no zeros. This completes the proof.

Acknowledgements. The first and the third authors are grateful to the Department of Mathematics of the University of Hong Kong for its warm hospitality during their visit when part of this work was done.

\section{References}

[1] S. S. Abhyankar, P. Eakin and W. J. Heinzer, On the uniqueness of the coefficient ring in a polynomial ring, J. Algebra 23 (1972), 310-342.

[2] S. S. Abhyankar and T.-T. Moh, Embeddings of the line in the plane, J. Reine Angew. Math. 276 (1975), 148-166.

[3] T. Asanuma, Non-linearizable algebraic $k^{*}$-actions on affine spaces, Invent. Math. 138 (1999), 281-306.

[4] P. M. Cohn, Free Rings and Their Relations, 2nd Ed., Academic Press, London, 1985. 
[5] W. Danielewski, On the cancellation problem and automorphism groups of affine algebraic varieties, preprint, Warsaw, 1989.

[6] A. van den Essen, Polynomial Automorphisms and the Jacobian Conjecture. Progress in Mathematics 190, Birkhäuser Verlag, Basel, 2000.

[7] K.-H. Fieseler, On complex affine surfaces with $\mathbf{C}^{+}$-action, Comment. Math. Helv. 69 (1994), 5-27.

[8] G. Freudenburg and L. Moser-Jauslin, Non-equivalent embeddings of Danielewski surfaces and non-linearizable actions of $\mathcal{O}(2)$ on $\mathbf{C}^{4}$, Math. Z., to appear.

[9] T. Fujita, On Zariski problem, Proc. Japan Acad. Ser. A Math. Sci. 55 (1979), 106-110.

[10] H. Kraft, Challenging problems on affine n-space, Astérisque 237 (1996), 295-317.

[11] R. Lyndon and P. Schupp, Combinatorial Group Theory. Reprint of the 1977 edition. Classics in Mathematics. Springer-Verlag, Berlin, 2001.

[12] M. Miyanishi, Some remarks on polynomial rings, Osaka J. Math. 10 (1973), 617-624.

[13] M. Miyanishi and T. Sugie, Affine surfaces containing cylinderlike open sets, J. Math. Kyoto Univ. 20 (1980), 11-42.

[14] R. Rentschler, Operations du groupe additif sur le plane affine, C. R. Acad. Sci. Paris 267 (1968), 384-387.

[15] I. P. Shestakov and U. U. Umirbaev, Poisson brackets and two-generated subalgebras of rings of polynomials, J. Amer. Math. Soc., to appear.

[16] V. Shpilrain and J.-T. Yu, Embeddings of hypersurfaces in affine spaces, J. Algebra 239 (2001), 161-173.

[17] V. Shpilrain and J.-T. Yu, Affine varieties with equivalent cylinders, J. Algebra 251 (2002), 295-307.

[18] D. Wright, On the Jacobian conjecture, Illinois J. Math. 25 (1981), 423-440.

L. Makar-Limanov

Department of Mathematics

Wayne State University

Detroit, MI 48202

USA

e-mail: lml@math.wayne.edu

\section{Shpilrain}

Department of Mathematics

The City College of New York

New York, NY 10031

USA

e-mail: shpil@groups.sci.ccny.cuny.edu http://www.sci.ccny.cuny.edu/ ${ }^{\sim}$ shpil/

(Received: October 14, 2002)

(Received: October 14, 2002)
P. van Rossum

Department of Mathematical Sciences

New Mexico State University

Las Cruces, NM 88011

USA

e-mail: petervr@nmsu.edu

J.-T. Yu

Department of Mathematics

The University of Hong Kong

Pokfulam Road

Hong Kong

e-mail: yujt@hkusua.hku.hk

http://hkumath.hku.hk/ jtyu 